\title{
DE LA PERSONALIDAD DOCENTE A LA METODOLOGÍA DE INVESTIGACIÓN: HISTORIZANDO LA FORMACIÓN DEL PROFESORADO BASADA EN LA INVESTIGACIÓN EN FINLANDIA
}

\section{From teacher personality to research methodology: Historicizing research-based teacher education in Finland}

\author{
Johanna Sitomaniemi-SAN \\ University of Oulu, Finland
}

Recibido: 23 de septiembre de 2020. Envío a informantes: I de octubre de 2020.

Aceptación definitiva: 5 de mayo de 202I

RESUMEN: Este artículo reexamina una premisa que se sostiene comúnmente en relación con el efecto de la formación, científica y basada en la investigación, del profesorado en Finlandia, la cual emergió en la década de i970. La premisa es que este nuevo tipo de formación produce un profesorado que es dirigido o disciplinado a través de la ciencia. En el centro se encuentra la manera en que construcciones históricamente específicas de «ciencia» alimentan las formas en las que el profesorado es concebido, fabricado y gobernado. En un esfuerzo por desestabilizar lo obvio de la «investigación» en la formación del profesorado en Finlandia, se piensa con la noción de alquimia elaborada por Popkewitz para analizar la racionalidad a través de la que se trabaja el ser docente. El análisis saca a relucir y explora detenidamente una conexión no reconocida previamente entre los tópicos de la «personalidad docente» y de la «ciencia-investigación», remitiendo a que la forma especulativo-vocacional no tiene por qué haber sido desplazada ni reemplazada necesariamente por la «nueva» forma científico-profesional de formar al profesorado.

PAlabRas Clave: Finlandia; formación de profesores; historizar.

AвSTRACT: This article re-examines a commonly held assumption about the effect of Finnish scientific, research-based teacher education, which emerged in the 
I970s; the assumption being that this newer mode of training produces teachers who are directed and/or disciplined through science. At issue is how historically specific constructions of «science» feed into the ways in which the teacher is envisioned, fabricated and governed. In an effort to destabilize the self-evidence of «research» in the present of teacher education in Finland, Popkewitz's notion of alchemy is thought with in order to consider the rationality through which the teacher's self is worked on. The analysis brings into view and scrutinizes a previously unrecognized connection between the motifs of «teacher personality» and «science-research», arguing that the speculative-vocational mode may not necessarily have been displaced and replaced by the «new» scientific-professional mode of teacher training.

KEY WORDS: Finland; Finlandeacher's training; historizing.

\section{Introducción}

Cada profesor [...] tiene su propia manera personal de relacionarse con su trabajo y con sus estudiantes. Los profesores serían privados de su mayor capacidad de influir [a sus estudiantes] si se les requiriese que abandonaran su originalidad. Desde la perspectiva de la educación, resultaría francamente aterrador si los profesores fuesen todos iguales. (Haavio, 1948, p. $\left.26^{*}\right)^{1}$

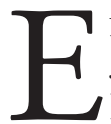
N UN LIBRO EDITADO en finés y publicado en 1999, La formación del profesorado en la ruptura de la Modernidad, el investigador educativo Hannu L. T. Heikkinen se pronuncia a favor del resurgimiento de la personalidad docente como una idea para la formación del profesorado en tiempos posmodernos. Para Heikkinen, la personalidad docente fue, tiempo atrás, un asunto central en la preparación del profesorado. Lo fue desde los tiempos de Uno Cygnaeus (I8Io-I888), 'padre' de la educación pública finlandesa, quien «enfatizó los requisitos para el profesorado en su condición de persona, insistiendo en la alta moralidad del profesorado» (Heikkinen, I999, p. I8I*), hasta los de Martti Haavio, cuyo libro, Personalidad docente (1948), se volvió un clásico para los estudiantes de formación del profesorado en las décadas de 1950 y 1960.

Heikkinen sugiere que este enfoque sobre la formación del profesorado, en el que la idea de la personalidad docente desempeñaba un papel esencial, fue «sepultado en la década de los 60 y los 70» (Heikkinen, I999, p. I81*), en la medida en que la formación finlandesa del profesorado fue transformada en un proyecto más científico. De hecho, durante esas décadas, a través de reformas específicas sobre la educación superior y la formación del profesorado, las formas vocacionales de la formación de los docentes dieron paso a enfoques basados en la Universidad, que enfatizaron la relevancia de la «ciencia» en la preparación del

El asterisco (*) indica que la cita textual ha sido traducida en dos ocasiones. En primer lugar, desde el finés al inglés por la autora del estudio. En segundo lugar, desde este último idioma al español por el traductor del texto. 
profesorado. Heikkinen percibe esta transición de lo «vocacional» a lo «científico» como pavimentadora del camino hacia un papel más técnico del profesorado. Para él, este papel fue influido por el giro hacia el positivismo en la investigación educativa, separando, consecuentemente, "conocimientos de valores» (Heikkinen, 1999, p. I82*) y que fue promovido aún más por la disolución de una cultura que había orientado la «base cristiano-patriótica» de la tarea educativa del profesorado (Heikkinen, 1999, p. I8*). Sin embargo, defiende Heikkinen, el «nuevo contexto social» (1999, p. $\left.18 \mathrm{I}^{*}\right)$ del posmodernismo parece indicar una nueva necesidad y una nueva oportunidad para restablecer la centralidad de la personalidad docente en la formación de los profesores. Es decir, Heikkinen percibe que, en una etapa definida por el relativismo, la información fragmentada y las ideas nómadas, el «profesorado posmoderno» debe trabajar aún más para involucrarse en la reflexión y en la formación de identidades con el objetivo de «conocerse a sí mismo» (Heikkinen, I999, p. 184*) y crear una «narrativa unificada» (Heikkinen, 1999, p. 189*). Para Heikkinen, tales proyectos funcionan como una invitación para reinstalar la noción olvidada y abandonada de la «personalidad docente». Entonces, la recuperación y el reavivamiento de la «personalidad docente» solicitados en el escrito de Heikkinen pueden ser leídos como un argumento contra el tecnicismo de las orientaciones científicas y como una invitación humanista (y quizás nostálgica) a restaurar tanto «valores» como la formación de un sentido de identidad coherente en la formación del profesorado.

En las literaturas sobre la formación finlandesa del profesorado, Heikkinen no se encuentra solo a la hora de hacer circular la narrativa histórica que asume una separación clara de su pasado vocacional a medida que lo «científico» se inserta en dicha formación. Es decir, ya sea percibido para mejor o para peor, la «ciencia» ha sido tomada seriamente en una variedad de relatos pedagógicos, sociológicos e históricos a la hora de discutir y debatir su efecto sobre los docentes en construcción. Además del tecnicismo expresado por Heikkinen, otros resultados percibidos de una formación del profesorado más científica varían desde unos docentes con vocación científica (Kansanen, 2007), al papel debilitado de la práctica (Säntti, Rantala, Salminen y Hansen, 20I4), la profesionalización científicamente legitimada del profesorado y de los formadores de docentes (Simola, Kivinen y Rinne, 1997), el currículum descontextualizado de la formación docente (Simola, 1998a) y la producción de un profesorado que ahora está «gobernado por la ciencia» (Saari, Salmela y Vilkkilä, 20I4, p. 194).

Sin embargo, mientras que las certezas, dudas y preocupaciones de cada relato pueden diferir, lo que se mantiene a lo largo de ellos es la «verdad» de la ciencia como una sustancia que, una vez insertada, ha llevado a algo que es «más científico» que antes. Al desestabilizar esta verdad, este artículo reexamina diferentes premisas que se mantienen comúnmente en relación con el efecto de la formación finlandesa del profesorado basada en la investigación, siendo estas premisas que esta nueva forma de formación produce docentes que están dirigidos o disciplinados mediante los «principios del pensamiento científico» (Saari et al., 2014, p. 194). Lo que se cuestiona es la manera en que construcciones históricamente específicas 


\section{DE LA PERSONALIDAD DOCENTE A LA METODOLOGÍA DE INVESTIGACIÓN: HISTORIZANDO 264 LA FORMACIÓN DEL PROFESORADO BASADA EN LA INVESTIGACIÓN EN FINLANDIA JOHANNA SITOMANIEMI-SAN}

de «ciencia» e «investigación» alimentan las formas en las que el profesorado es concebido, fabricado y gobernado en el presente. Al emplear citas alarmistas sobre la ciencia y la investigación se busca enfatizar este enfoque crítico: en lugar de tomar esas nociones como prácticas universales, examino «ciencia» e «investigación» como construcciones que contribuyen a una racionalidad cultural e históricamente específica que es aplicada a la preparación del profesorado.

Específicamente, el análisis que se desarrolla saca a la vista y escudriña una conexión previamente no reconocida entre los tópicos de la «personalidad docente» y de la «ciencia-investigación» en la formación finlandesa del profesorado. Si bien es frecuente suponer (como en el relato de Heikkinen del que he hablado anteriormente) que la «personalidad docente» representa al espíritu vocacional de la formación docente que hace mucho tiempo fue reemplazado por un ethos mucho más científico y racional, también es posible argumentar que los dos proyectos aparentemente opuestos de formación del profesorado no se excluyen necesariamente entre ellos. De hecho, como defiendo en este artículo, la introducción de la ciencia-investigación, al reconfigurar los discursos sobre la formación del profesorado, puede ser percibida como aquello que preserva la tarea de trabajar en la personalidad, la individualidad y la moralidad del profesorado.

En este artículo, entonces, revisito diferentes presencias y ausencias específicas, así como rupturas y continuidades que han sido identificadas y explicadas previamente en la literatura sobre la formación finlandesa del profesorado y sobre su historia. En la literatura, la reforma de la década de 1970, a través de la cual la formación docente finlandesa se unificó como un programa académico y basado en la universidad, se ha convertido en una línea divisoria bien establecida entre la formación del profesorado «vieja» y la «nueva», entre lo «vocacional» y lo «científico» y entre lo que una vez «fue» y ahora «es». Sin embargo, un enfoque historicista y genealógico (Foucault, 1991; Popkewitz, Franklin y Pereyra, 200I; Popkewitz, 20I3) desestabiliza esta línea divisoria fija, sugiriendo que lo que una vez fue todavía es efectivo. De esta manera, como característica de las genealogías, la historización ofrece una manera de repensar tanto el presente como el pasado. Además, ofrece una manera de desmantelar la obviedad actual de la formación finlandesa del profesorado basada en la investigación como un principio curricular-pedagógico y, a la vez, de reexaminar la historia de su surgimiento de una forma que no asuma linealidad, causalidad u origen.

El artículo se centra en el caso específico de la formación del profesorado en Finlandia, aunque la relevancia y las implicaciones de los resultados se extienden más allá. Si bien la formación del profesorado científica y basada en la investigación se ha convertido en un componente importante de la marca internacional de la educación finlandesa (p. ej. Jakku-Sihvonen y Niemi, 2006b; Sahlberg, 2015) y, como tal, en un modelo y una referencia para las reformas de la formación del profesorado en otros lugares (p. ej., Darling-Hammond, 20I7), el problema de la ciencia y la investigación en la formación del profesorado no es, de ninguna manera, exclusivo del contexto finlandés. Por ejemplo, la emergencia de la «ciencia de la formación» en la década de 1960 en los EE. UU. (véase Labaree, 1992), así 
como de una variedad de movimientos (anglohablantes) de investigación docente desde la década de 1950 (véase Hammersley, 1993), apunta a unas trayectorias transnacionales e históricas más amplias en las que se embrolla el caso de formación docente finlandesa. Con todo, la especificidad histórico-cultural del paisaje finlandés de la educación (del profesorado) es importante: el «lenguaje de la educación» finlandés está influenciado por el protestantismo luterano (véase Tröhler, 20II) y está profundamente conectado con los ideales hegelianos de la patria nación y la tradición alemana de la bildung (véase Koski, 2005; Koski y Filander, 2013; Saari et al., 20I4). Entonces, atender al caso particular de Finlandia es prestar atención a cómo nociones y prácticas viajeras y circulantes a nivel transnacional, como las de ciencia e investigación (docente), vienen a tener sentido y tienen efectos en un espacio-tiempo específico.

El resto de este capítulo se encuentra dividido en cinco secciones. La primera introduce los debates sobre las reformas de los años 70 que abogaban por la inserción de la ciencia en la formación finlandesa del profesorado. La segunda sección revisa los relatos históricos anteriores presentes en la literatura y sugiere un replanteamiento de la cientificización y el disciplinamiento de la enseñanza y de la formación del profesorado, utilizando la noción de alquimia de Thomas Popkewitz. La tercera sección examina la noción de la personalidad docente en la manera en que es articulada en el discurso de la tradición vocacional y especulativa, mientras que la cuarta sección se centra en la reconfiguración del docente y de la «personalidad docente» a través de la construcción alquímica de la ciencia-investigación. El capítulo concluye analizando cómo la «alquimia de la investigación» pone de manifiesto una historia diferente de la formación finlandesa del profesorado basada en la investigación, desafiando así la suposición aparentemente obvia de que la formación de los docentes como «investigadores» y «expertos científicos» ha coincidido necesariamente con el desplazamiento de la noción vocacional de la personalidad docente ${ }^{2}$.

\section{La inserción de la ciencia en la formación finlandesa del profesorado}

Las reformas de la formación finlandesa del profesorado de la década de 1970 procuraron el traspaso de la formación del maestro de escuela primaria desde los seminarios docentes vocacionales hacia las universidades, así como a la empresa de la formación del profesorado de escuela secundaria. La reforma tuvo dos etapas. Primero, en 1974, después de una breve fase de transición, los seminarios docentes vocacionales fueron abolidos y la formación de los maestros de primaria comenzó como un programa de licenciatura en unidades de formación del profesorado dentro de las universidades. En 1979, la licenciatura de tres años sería transformada en una maestría de cinco años. Este último cambio fue influenciado

Este capítulo se deriva parcialmente de una tesis doctoral (SITOMANIEMI-SAN, 20I5). 
por la reforma general de la educación superior, que abolió los títulos de licenciatura en la educación superior finlandesa (Simola, 1993).

Las reformas que tuvieron lugar en la década de 1970 estuvieron precedidas de muchos años de debate sobre la reorganización de la formación del profesorado. Las preocupaciones en torno a la promoción de una forma más académica de formación docente fueron múltiples. Se consideraba que, después de su formación básica, los docentes deberían tener las cualificaciones que les habilitasen para continuar otros estudios académicos (Koskenniemi, I962)4. Además, se planteó una preocupación sobre la creciente brecha percibida en la calidad entre las escuelas urbanas y rurales: los maestros con antecedentes culturales y educativos «más ricos» buscaban una formación docente que los llevara a enseñar en las ciudades, mientras que las escuelas del área rural tenían que conformarse con los maestros «menos talentosos» y menos formados (Kyöstiö, 1965). De esta manera, la unificación de la formación del profesorado fue presentada como la solución a lo que se consideraba el problema de la distribución desigual de la calidad del profesorado en las escuelas finlandesas.

De manera más destacada, los esfuerzos para unificar y «elevar el nivel» de la formación docente finlandesa se engarzaron con el aumento de la investigación empírica en las ciencias educativas. Esta investigación vio fortalecida su posición en el pensamiento educativo finlandés después de la Segunda Guerra Mundial (véase Saari, 20II; Saari et al., 20I4). Esto es, el esfuerzo por reformar la formación del profesorado surgió de la visión que los investigadores educativos empíricos tenían sobre la enseñanza, la cual consideraban una práctica que debía ser informada e investigada a través del método científico. El currículum de la formación del profesorado debía basarse en el conocimiento producido científicamente, que prepararía a los futuros profesores entrenándolos para que comprendieran y trabajaran de acuerdo con las «leyes naturales» de la enseñanza (Koskenniemi, 1962).

Por tanto, las bases para la existencia de diferentes tipos de programas de capacitación del profesorado ya no parecían plausibles. Independientemente del tipo de escuela para la que se debía formar al profesorado para enseñars, lo que se

SimOla y RinNe (20IO) han señalado la contingencia de esta transformación de las licenciaturas en maestrías y han sugerido que, sin la reforma general, la formación del profesorado de primaria a través de las maestrías no tendría por qué haber comenzado. Debido al proceso europeo de Bolonia, referente a los títulos de educación superior, el sistema de dos niveles con títulos de licenciatura se reintrodujo en la formación finlandesa del profesorado (y en otros programas de títulos universitarios) a comienzos de los 2000. Los futuros docentes, sin embargo, continúan cursando los programas de formación del profesorado que conducen a títulos de maestría (véase JAKKU-SIHVONEN y NIEMI, 2006a).

4 Esta demanda no era nueva. Por ejemplo, Simola señala que «ya en I890, los maestros de primaria habían afirmado que su formación extendida debería organizarse a nivel universitario» (SimOla, I993, p. 172).

Hasta la década de 1970, el sistema educativo finlandés se basaba en un modelo de dos niveles que se fundamentaba en la estructura social finlandesa del siglo XIX. Los dos niveles consistían en el kansakoulu (inicialmente destinado a los plebeyos y que se refería a la escuela primaria; traducido literalmente como «escuela popular») y el oppikoulu (una especie grammar school o escuela secun- 
percibía que los futuros docentes tenían en común era la necesidad de un tipo de formación más científico. A partir de este argumento, se sugirió que una forma unificada de formación del profesorado se beneficiaría más de una ubicación en una facultad universitaria que de una ubicación en una institución propia y separada y, por lo tanto, de permanecer desligada de la universidad. Es decir, si la formación del profesorado se llevara a cabo en facultades pedagógicas dentro de las universidades:

[esta] estaría científicamente preparada en todos los aspectos y bajo supervisión científica. [...] La enseñanza en los programas de formación del profesorado estaría acompañada, apoyada y desarrollada continuamente por una amplia investigación científica. (Koskenniemi, 1962, p. 51*)

Dentro de esta línea de pensamiento, la formación del profesorado se percibía no solo de naturaleza pedagógica, sino también científica:

Lo que implica la preparación [...] del profesorado [...] es la supervisión en la competencia docente o, en otras palabras, la formación pedagógica. Se percibe que los profesores necesitan dicha formación para guiar el proceso de enseñanza, de una manera efectiva, dentro de las áreas de conocimiento y habilidades de las que son responsables. [...] La formación profesional, entendida de esta manera, implica familiarizarse con la teoría educativa, las premisas educativas y la realidad educativa. (Kyöstiö, 1965, p. $5^{*}$ )

En este cambio hacia la formación científica del profesorado, el «amateurismo» y el «diletantismo» (Kyöstiö, 1965, p. 9*) de los modos no académicos de formación del profesorado ${ }^{6}$ debían ser reemplazados por formas de conocimiento que fueron consideradas más científicas y sistemáticas. Específicamente, se consideraba que se debía generar «teoría educativa» y que las «premisas» y la «realidad» de la educación debían hacerse visibles a través de la investigación empírica más que a través de formas de conocimiento experienciales o especulativas. La formación del profesorado debía reformarse y «actualizarse» con un currículum más científico que permitiesen al profesor desprenderse del papel de un «técnico» (Kyöstiö, 1965, p. $8^{*}$ ) y pasar al de un profesional autónomo. En resumen, el ethos

daria inferior, a través de la cual se obtenía el acceso a la educación superior). Este sistema escolar paralelo fue abolido mediante el Sistema Escolar Comprehensivo, que se implementó durante la década de 1970 y que introdujo una ruta educativa obligatoria de nueve años para todo el alumnado (véase Simola, 1993).

6 Irónicamente, esta crítica no deja de estar presente tras la reforma de la formación del profesorado de la década de los 70. Consideremos el argumento de Kivinen y Rinne: «Lo que se ha recogido dentro de la formación del profesorado es una multitud de bric-a-brac; la repetición inútil del conocimiento general; las actividades obligatorias de música, manualidades y ejercicio físico [...] y [...] la didáctica que carece de contenido y que podría ser abandonada sin el menor atisbo de una catástrofe intelectual» (KIVINEN y RiNnE, I989, p. 419*). 
especulativo-vocacional debía ser reemplazado por un ethos científico-profesional que supuestamente acarrearía una verdadera autonomía para el profesorado.

Estos argumentos prevalecen en las décadas posteriores a las reformas de los años 70, situando a la nueva formación del profesorado científica, académica y basada en la investigación como opuesta a la antigua tradición de formación del profesorado vocacional y especulativa. En ese momento, se sugiere que el profesorado debería ser dotado de una «imagen científicamente confiable de los procesos de crecimiento y educación» y que «la filosofía no será de ayuda» (Viljanen, I988, p. $26^{*}$ ) para obtener dicho conocimiento. Los futuros docentes son imaginados como involucrándose en el pensamiento basado en la investigación cuando leen literatura científica y, por lo tanto, que «aprendan a debatir y argumentar con una referencia constante a la investigación, y sin depender del pensamiento cotidiano y los argumentos “mágicos” o “místicos” (Westbury, Hansén, Kansanen y Björkvist, 2005, p. 479).

Los argumentos a favor del profesorado basado en investigaciones científicas subrayan una nueva era en la que tanto la educación como la sociedad en general han entrado:

Es inútil reflexionar sobre si la formación impartida en el antiguo seminario fue mejor que la actual formación del profesorado de clase académica. Con todo el debido respeto a la bella tradición del seminario, el momento histórico es ahora diferente: el mundo también es un lugar diferente en este momento. ¡La formación docente debería poder autoorientarse bien en el siglo xxı! En pocas palabras, el objetivo de la formación del profesorado, en su forma actual, es la formación de docentes que tienen suficiente conocimiento basado en la investigación científica sobre el alumnado y su desarrollo; que tienen un buen dominio del conocimiento del contenido disciplinario que enseñar; que comprenden las demandas que los cambios en la sociedad imponen a la escuela; y que dominen suficientemente las habilidades básicas requeridas para guiar el proceso de enseñanza. (Uusikylä, I992, p. $\left.58^{*}\right)$

En tales argumentos, se considera que el profesorado científicamente informado y con pensamiento científico estará mejor preparado para los desafíos y para la sociedad del futuro; mejor preparado para educar a los ciudadanos venideros para una sociedad rápidamente cambiante (véase Saari, 2013). La inserción de la investigación y el conocimiento científicos en la formación del profesorado viene a marcar la transición del pasado, definido por la tradición y la ignorancia, a una vía de progreso más racional, en la que la filosofía es desplazada hacia los márgenes debido a su dogmatismo y a su naturaleza especulativa (véase Saari, 20II; Saari et al., 20I4). En tales cambios, se supone que los supuestos obsolescencia y atraso de la formación del profesorado son dejados atrás en el espíritu de la Ilustración: la ciencia viene a significar el progreso y el futuro abierto de posibilidades (ver Popkewitz, 2005). 

JOHANNA SITOMANIEMI-SAN

\section{Repensando la cientifización y el disciplinamiento de la formación del profesorado y de los docentes}

En la literatura pedagógica sobre la formación docente finlandesa, la cientificización de la formación del profesorado es asumida como uno de los factores históricos influyentes más importantes en sus propiedades actuales, así como en su calidad. Como resultado de las reformas de los 70, la presencia de la ciencia y de la investigación en la formación del profesorado es percibida como posibilitadora de un currículum que combina los objetivos de competencia en pensamiento científico y en habilidades de investigación, con la aptitud pedagógica. Se sugiere que el currículum de la formación del profesorado basada en la investigación contribuye a la formación de docentes científicamente letrados, pedagógicamente informados por el conocimiento investigado y capaces de «fusionar»o «integrar» teoría y práctica (p. ej., Maaranen y Krokfors, 2008; Silander y Välijärvi, 2013).

En el discurso pedagógico, la noción del profesorado como investigador es referida como un producto evidente del currículum. Se enfatizan las características del profesor que es hecho posible a través de la formación docente basada en la investigación: «La ambición no es preparar investigadores profesionales, sino cualificar a profesores capaces de trabajar como profesionales autónomos, responsables y reflexivos» (Hansén y Eklund, 20I4, p. 5). Sin embargo, aunque la mayoría de los relatos apoyan esta narrativa, la orientación científica de la formación del profesorado es considerada por algunos como potencialmente perjudicial. Se han planteado inquietudes sobre los efectos del enfoque basado en la investigación en el «contexto escolar real» (Säntti et al., 20I4, p. 35), sugiriendo que el énfasis en la investigación en la formación del profesorado no contribuye necesariamente al trabajo práctico de los docentes de una forma relevante o efectiva (véase también Hansén y Eklund, 20I4; Kivinen y Rinne, 1989).

Desde una perspectiva diferente, histórico-sociológica, la cientificización de la formación finlandesa del profesorado ha sido examinada y vista como un «disciplinamiento» de la base de conocimientos de la formación del profesorado y de los propios docentes. El trabajo de Hannu Simola sobre los discursos educativos estatales identifica un cambio particular que tuvo lugar a finales de la década de 1960:

Una combinación múltiple, pragmática e ideológica, de conocimiento de contenido ético, psicológico, pedagógico, histórico [...] fue reemplazada por la «nueva» verdad, mediante la cual una ciencia educativa orientada didácticamente da forma a la base de conocimiento para el trabajo de los profesores. (Simola, I998b, p. 329)

Desde este punto de vista, la didáctica ${ }^{7}$ emerge como una nueva «ciencia de la enseñanza», que, a través de sus enfoques experimentales en la psicología edu-

El término «didáctica» debe ser entendido aquí como influenciado por la tradición germana de la Bildung-Didaktik (véase e. g. Autio, 2006, 20i4; Westbury, Hopmann y RiQuarts, 2000). 
DE LA PERSONALIDAD DOCENTE A LA METODOLOGÍA DE INVESTIGACIÓN: HISTORIZANDO

cativa (Simola et al., 1997; Simola, 1998a; Simola, 1998b), reorienta la formación finlandesa del profesorado desde un «ethos vocacional tradicional hacia un profesionalismo más racionalizado y legitimado por la ciencia» (Simola, 1993, p. 168). El disciplinamiento, en el argumento de Simola, apunta a cómo los enfoques de la investigación educativa orientados histórica y filosóficamente son marginados a medida que los enfoques científicos (predominantemente angloamericanos) de la investigación educativa se apoderan del campo. A través de este cambio, el conocimiento del «profesor "disciplinado"» (Simola, 1998c, p. 736) viene a ser definido y limitado a lo que se produce científicamente y, por lo tanto, es considerado como «verdadero» 8 .

$\mathrm{Al}$ revisar esta aparición del conocimiento científico y didáctico en el campo de la investigación educativa, el análisis de Saari et al. (2014) sobre el discurso curricular finlandés ofrece, sin embargo, otra visión sobre el supuesto efecto que tiene la inserción de la ciencia en la formación del profesorado. Desde su postura foucaultiana, estos autores perciben este disciplinamiento como una forma de gobierno, ya que los maestros son formados para adoptar una mirada científica y disciplinada y "pensar en sí mismos como profesionales científicos autónomos» (Saari et al., 2014, p. 194). Por lo tanto, sugieren que:

El disciplinamiento se lleva a cabo dentro de las mentes de los maestros, como su subyugación voluntaria a los principios del pensamiento científico. En términos foucaultianos, podríamos pensar en esta forma de subjetividad como un «soberano esclavizado» [Foucault, 1966, p. 323] -un sujeto conocedor que es libre en la medida en que lo rige la ciencia-. (Saari et al., 20I4, p. 194)

Si bien estas diferentes narrativas históricas discutidas anteriormente varían en perspectiva, parecen compartir un supuesto sobre la aparente existencia de la «ciencia» como una cosa o una sustancia de lógica pura que es insertada en la formación del profesorado -ya sea con el efecto percibido de producir un profesor científicamente informado, una visión más disciplinada del conocimiento del docente o una materia docente gobernada por la ciencia-.

Sin embargo, la noción de alquimia, de la manera en que es desarrollada en el trabajo de Thomas Popkewitz, desestabiliza este supuesto de la ciencia como algo que debe tomarse al pie de la letra en su inserción en la formación finlandesa del profesorado. En los estudios de Popkewitz sobre la reforma escolar (p. ej., 1998, 2002, 2007, 2010), la alquimia es una metáfora a través de la cual se puede considerar cómo las disciplinas académicas (como la historia, las matemáticas o la música) se transforman en asignaturas escolares que llevan los mismos nombres, pero, sin embargo, «haciendo» algo diferente. La alquimia llama la atención sobre cómo las asignaturas escolares inscriben visiones sociales, psicológicas y pedagógicas, sobre quién debería ser el niño o el aprendiz, produciendo así ideales sobre

El enfoque sociológico de Simola examina el surgimiento de una «ciencia de la enseñanza» principalmente como una cuestión relacionada con la credibilidad académica y el estatus profesional de los formadores de profesores. 
el individuo autónomo, como, por ejemplo, un solucionador de problemas o un tomador de decisiones:

Los estándares del currículo nacional en música versan, fundamentalmente, sobre la capacidad del niño para pensar (tomar decisiones informadas o resolver problemas), desarrollar habilidades de comunicación (defender un argumento, trabajar eficazmente en grupos), producir un trabajo de calidad (adquirir y usar información), y hacer conexiones con la comunidad (reconocer y actuar sobre la base de las responsabilidades como ciudadano). [...] ¡Las materias escolares son transformadas así en representaciones de las psicologías del niño y del profesor! (Popkewitz, 2002, pp. 262-263)

Popkewitz argumenta que, a través de la alquimia, las materias escolares se transforman «en reglas y estándares morales diferenciales para la acción y la participación» (Popkewitz, 2007, p. 78); en prácticas a través de las cuales se administran y gobiernan «las disposiciones, sensibilidades y conciencias del niño» (Popkewitz, 2002, p. 262).

Por lo tanto, la noción de alquimia ofrece una forma, inexplorada previamente, de dar sentido a la cientificización de la formación finlandesa del profesorado. Lo que hace posible es repensar cómo la «ciencia» contribuye a la producción del (futuro) profesor, en la medida en que esta se transforma en una racionalidad para gobernar su autonomía (del profesor). Al considerar la formación del profesorado basada en la investigación como un lugar en el que se dirigen y regulan las disposiciones y las sensibilidades del docente a través de la alquimia de la investigación, resulta, por un lado, menos evidente asumir una historia lineal y evolutiva en la que la inserción de la ciencia durante las reformas de la década de 1970 representa el punto de origen. Por otro lado, al pensar con la noción de alquimia de Popkewitz, también es posible diversificar, añadir matices y desafiar los relatos ofrecidos por Simola, Saari y sus colegas sobre la cuestión del «disciplinamiento» a través de la ciencia en el caso de la formación finlandesa del profesorado.

\section{La personalidad docente y el ethos vocacional}

En cierto sentido, la distinción hecha en la literatura pedagógica entre los modos vocacionales y científicos de la formación del profesorado se relaciona con las dicotomías de práctica-teoría y de práctica-investigación. En un número de 1977 de Kasvatus (Educación, en español), la revista finlandesa líder en el campo de la educación, se puede encontrar un buen ejemplo de ello durante las reformas de la formación del profesorado. En este número, se realiza un breve intercambio de ideas entre dos investigadores educativos, Martti Kaikkonen y Juhani Hytönen, sobre el propósito y el foco de la formación del profesorado. El argumento central de Kaikkonen es que los profesores «deben saber cómo hacer» (Kaikkonen, I977, p. I05), proyectando que la nueva presencia de métodos de investigación 
DE LA PERSONALIDAD DOCENTE A LA METODOLOGÍA DE INVESTIGACIÓN: HISTORIZANDO

en el currículum puede ser de poca relevancia práctica para los profesores, cuya principal preocupación es saber cómo enseñar y educar. Hytönen, por otro lado, responde a Kaikkonen con su propio contraargumento de que los profesores «deben saber cómo pensar» (Hytönen, 1977, p. I07, énfasis añadido) y sugiriendo que la inserción de los métodos de investigación en el currículum de la formación del profesorado es precisamente lo que mejorará las habilidades profesionales del profesor, ya que le proporciona herramientas metodológicas con las cuales pensar y hacer observaciones?.

Sin embargo, en otro archivo, aunque relacionado con lo anterior, la distinción entre los modos vocacionales y científicos de formación del profesorado hace referencia a las diferentes formas disciplinarias de razonar sobre quién debería ser el profesor. La escritura de Martti Haavio, un «representante eminente» (Simola, I993, p. I76) de la antigua tradición del seminario, como lo atestiguan tanto él (Haavio, 1965) como otros (Heikkinen, 1999; Simola, 1993; Viljanen, 1979), proporciona un útil ejemplo. El libro de Haavio, Personalidad docente, publicado por primera vez en 1948 y reimpreso varias veces a partir de entonces, encarna el enfoque especulativo - un enfoque que produce lo que Simola (1993) reseña como el «ethos misionero» de la formación vocacional del profesorado, con un énfasis en la llamada (en un sentido vocacional) que experimenta el profesor como educador-. Específicamente, el enfoque especulativo de la Personalidad docente de Haavio resuena con la tradición educativa alemana de la Geisteswissenschaftliche Pädagogik. Esto resulta evidente no solo en las referencias explícitas del texto a escritores dentro de esta tradición (como Kerschensteiner), sino también en la forma asociada de escritura programática que presenta, así como en su énfasis en (el cultivo de) el ser interior de la personalidad, o condición de persona ${ }^{\mathrm{I}}$.

En su mayor parte, Personalidad docente se centra en el «ser pedagógico del profesor», discutiendo en detalle una variedad de cualidades que son "comúnmente requeridas por un buen profesor» (Haavio, 1948, p. $25^{*}$ ) y que, juntas, se percibe que componen una «figura docente básica» (Haavio, I948, p. $25^{*}$ ). Haavio explora estas cualidades -que sugiere que son psicológicas, éticas y didácticas en naturaleza- a través de las siguientes áreas: autoridad y disciplina en el aula, comprensión de la individualidad del estudiante, tacto pedagógico, vida intelectual, vida estética, amor pedagógico, paciencia, justicia, humildad, sinceridad, vocación, alegría, sentido de responsabilidad, el profesor como modelo moral que seguir $\mathrm{y}$, por último, la trascendencia de la fe cristiana personal del profesor ${ }^{\mathrm{II}}$. Como tal, y a través de una forma programática de escritura, la exploración que

Véase SäNTTi et al. (20I4) para un ejemplo de expresiones actuales y continuadas de preocupación a este respecto.

1o Véase Horlacher (2016) en relación a la centralidad de la personalidad del educador y Tröhler (2003) en relación a la noción alemana de Persönlicbkei influenciada por la Bildung.

II Este lenguaje abiertamente religioso no es inusual en el tiempo del escrito de Haavio. Véase el estudio de KOSKI (2005) sobre los libros finlandeses de ABC que examina el cambio desde el cristiano luterano a los principios secularizados para la moralidad y la educación moral en la sociedad finlandesa durante la década de 1960. 
realiza Haavio sobre los ideales docentes es atribuida a «la identidad vocacional tradicional de los profesores, [...] basada en un espíritu luterano y patriótico» (Simola, 1993, p. 173).

Sin embargo, mientras ofrece estas cualidades como ideales para el profesorado, Haavio señala que estas aparecen de una manera única en cada profesor:

Cada profesor [...] tiene su propia manera personal de relacionarse con su trabajo y con sus estudiantes. Los profesores serían privados de su mayor capacidad de influir [a sus estudiantes] si se les requiriese que abandonaran su originalidad. Desde la perspectiva de la educación, resultaría francamente aterrador si los profesores fuesen todos iguales. (Haavio, 1948, p. 26*)

El contemporáneo de Haavio J. E. Salomaa, en un artículo titulado «Sobre el ser del educador», elabora, de una manera similar, esta noción de la personalidad individual del profesorado:

Con independencia de todas las cosas diferentes que se requieren del profesor, lo más determinante es que él/ella es una personalidad, una persona. La conjunción de persona y profesión en el profesor significa que cada profesor es un individuo. El «maestro de escuela», en el sentido negativo del término, implica el tipo profesional que se establece en sus maneras, un extraño a la vida, de mente estrecha, pedante, sin espíritu. Tal profesor no logrará, incluso con la mejor voluntad del mundo, resultados educativos significativos. Los resultados solo serán alcanzados por el profesor que influye en los estudiantes a través del poder sincero y vehemente de su personalidad individual. (Salomaa, 1947, p. $5^{*}$ )

En estas expresiones, el propósito percibido de la formación del profesorado es el cultivo, y el autocultivo, de la personalidad y el carácter del profesor. Esta forma de razonamiento especulativo y hermenéutico influenciado por la bildung es lo que está en el corazón del ethos vocacional: la formación del profesorado y el cultivo de las cualidades y disposiciones del profesor dentro de los órdenes morales de la nación. La personalidad del docente, entonces, es un tema específico que está inscrito en el discurso asociado con la tradición del seminario vocacional y que está incrustado en la figura de la «vela de la nación». Esta es la noción de la personalidad del docente que está aparentemente desplazada en el nuevo tipo científico de formación del profesorado: se considera que el enfoque especulativo, como es evidente en las cavilaciones de Haavio sobre la personalidad del docente, produce "“novelas pedagógicas» en lugar de contribuciones científicas» (Kähkönen, 1979, p. I30, según lo citado y parafraseado por Simola, 1993, p. 176). Para los defensores del nuevo enfoque científico de la formación del profesorado, tanto los ideales docentes definidos como las formas asociadas de investigación representadas por académicos como Haavio son considerados obsoletos e insuficientes para los requisitos de la formación del profesorado y la enseñanza modernas (Viljanen, 1979). 
DE LA PERSONALIDAD DOCENTE A LA METODOLOGÍA DE INVESTIGACIÓN: HISTORIZANDO 274 LA FORMACIÓN DEL PROFESORADO BASADA EN LA INVESTIGACIÓN EN FINLANDIA JOHANNA SITOMANIEMI-SAN

\section{La metodología de investigación y el etbos científico}

A medida que la educación orientada empíricamente «se imponía» (Saari et al., 20I4, p. 192) durante los años posteriores a la Segunda Guerra Mundial, el razonamiento disciplinario sobre la formación del profesorado y el profesor cambió. Si bien los enfoques filosóficos e históricos habían sido previamente el medio principal a través del cual investigar las cuestiones de la enseñanza y de la formación del maestro, estas formas de investigación estaban ahora marginadas por el nuevo dominio de los enfoques empíricos. Se volvió lógicamente evidente que los procesos y prácticas de enseñanza debían ser estudiados a través del método científico empírico. Además, el nuevo argumento concomitante era que los profesores no solo debían familiarizarse con el conocimiento producido a través de dicho método, sino también con el método en sí mismo.

Esta línea de pensamiento está presente con mayor fuerza en el trabajo del investigador empírico educativo finlandés Matti Koskenniemi y sus colegas. El proyecto de investigación más importante de Koskenniemi, DPA Helsinki (19671977), en el que la abreviatura significa «análisis didáctico de proceso», consistía en producir conocimiento sistemático sobre el proceso de enseñanza utilizando métodos de investigación empírica. A través de una variedad de aparatos de observación, incluidos espejos unidireccionales y cámaras de vídeo (p. ej., Komulainen, 2009; Uusikylä, 1980), los «patrones», «variables» y «regularidades» del evento de enseñanza dentro del contexto del aula se convirtieron en el objeto de la mirada del investigador. En particular, mediante el uso de taxonomías de observación (véase Saari, 20II), debían hacerse visibles, observables y analizables las características y factores del evento de enseñanza, incluyendo aspectos como las propiedades de la comunicación verbal, la atmósfera emocional en el aula, las relaciones de autoridad, la flexibilidad del profesor y las características de la participación del estudiante (Uusikylä, 1977).

A través de este modo de razonamiento koskenniemiano y DPA, emerge una nueva racionalidad, construyendo al profesorado como un tipo específico de investigador-científico. Aquí, la metodología de investigación empírica se crea como una competencia central no solo para el investigador, sino también para el profesor. Al aprender a utilizar metodologías y aparatos de investigación, se percibe el proceso de enseñanza en toda su complejidad como volviéndose visible, observable y analizable para el profesor -al igual que para el científico-. Sin embargo, en esta racionalidad emergente, lo que es central para la creación y el gobierno del profesor no es el «objeto empírico» como tal, sino las prácticas del método empírico. La competencia en la metodología de investigación es concebida como provisora de los medios para analizar momentos y eventos específicos en el aula al profesor, para que este luego pueda ejercer su propio juicio y pensamiento independiente -este es el «pensamiento científico» que se concibe que adopta el profesorado-. Para los investigadores con mentalidad DPA, los profesores que tienen herramientas metodológicas de investigación a su disposición están mejor equipados para tomar «decisiones personales» en el aula. Estos 
profesores dependerán menos de recetas estandarizadas de «cómo hacer» para enseñar (Uusikylä, 1977, p. 87\%). De esta manera, la «investigación científica» se inserta como una tecnología a través de la cual el profesorado debe ser liberado de cualquier tipo de tecnicismo y dogma, ya sea definido por la rutina, la tradición o la teoría (Koskenniemi, I966; Malinen, 1974b; Viljanen, 1979).

Es dentro de esta racionalidad donde la alquimia de la investigación se torna evidente. "Investigación» o "pensamiento científico»-que en el vocabulario del DPA también recibe el nombre de "pensamiento didáctico»- se transfiguran en reglas morales y pedagógicas para el pensamiento, la acción y las disposiciones del profesor. La noción de investigación se transforma en instrucciones sobre quién debería ser el profesor autónomo y tiene poco que ver con lo que la «investigación» implica como práctica científica y disciplinaria. Es decir, la noción de investigación, junto con sus nociones sinónimas de pensamiento científico y didáctico, llega a fabricar a un profesor-investigador-científico que debe ser consciente y creativo en su pensamiento y acción, un profesor que está atento a las situaciones únicas que surjan en el aula y responsable de sus decisiones y soluciones en estas situaciones:

Según el pensamiento didáctico, [el seguimiento de] instrucciones detalladas deben ser evitadas. En cambio, el objetivo para el profesor es que, en cada situación, considere qué curso de acción contribuiría mejor al desarrollo del alumno. (Koskenniemi y Hälinen, 1970, p. 248*)

El profesor debe actuar de una manera basada en el conocimiento investigado y científico. La aplicación de la investigación requiere de una interpretación continua de los objetivos educativos y de la elección de diferentes métodos educativos. El conocimiento establecido nunca está disponible para tales situaciones; el pensamiento creativo y la interpretación independiente del profesor son necesarios. (Malinen, 1974b, p. 16*)

De esta manera, se concibe que el pensamiento científico-didáctico emancipa al profesor de la dependencia del hábito, la rutina y la autoridad externa y lo conduce hacia el pensamiento y la acción autónomos, aumentando así su capacidad (del profesor) para asumir responsabilidad personal profesional y responder al propósito moral de la enseñanza. El profesor con «pensamiento científico-didáctico» debe comprender la naturaleza contextualmente específica de cada situación durante el evento de enseñanza, así como que la solución a un problema es dependiente de una conciencia y evaluación de ese contexto específico, mientras se mantienen en mente la dirección educativa y el propósito de la enseñanza. Dentro de la racionalidad koskenniemiana, el pensamiento científico-didáctico es el que (finalmente) traerá verdadera autonomía y los profesores llevarán a cabo tal pensamiento a través de su personalidad docente:

El profesor puede guiar y apoyar el crecimiento de sus estudiantes hacia la condición de persona siempre y cuando que ella/él opere como una personalidad en 
DE LA PERSONALIDAD DOCENTE A LA METODOLOGÍA DE INVESTIGACIÓN: HISTORIZANDO

276 LA FORMACIÓN DEL PROFESORADO BASADA EN LA INVESTIGACIÓN EN FINLANDIA

JOHANNA SITOMANIEMI-SAN

sí misma/o. Esto solo es posible si ella/él conoce las leyes naturales ${ }^{12}$ del evento de enseñanza, analiza y evalúa los eventos ante ella/él [...], en otras palabras, si ella/él piensa didácticamente. (Koskenniemi, 1968, pp. 223-224)

Surge una doble ocupación en el posicionamiento del profesor como alguien que se pone en el lugar del investigador para ver y analizar el evento de enseñanza. Por un lado, los cursos de formación del profesorado en metodología de investigación tienen como objetivo dirigir al profesor hacia una mirada científica en el aula. Por otro lado, en las prácticas de enseñanza, esa mirada científica se dirige hacia el ser del profesor.

Es muy natural que cada estudiante para profesor, [...] a través de un televisor interno, reciba comentarios sobre su propia enseñanza, que luego, junto con los supervisores, pueden ser analizados dentro del marco de la didáctica general, así como pueden basarse en la investigación sobre el evento de enseñanza. Es una cuestión de formar a los profesores como profesionales que deben resolver los problemas complejos que surgen en el proceso de enseñanza. (Alikoski, 1973, p. 214*)

A través de la autoobservación, de la manera en que es dirigida por la mirada proporcionada por las herramientas de la metodología de investigación (Hytönen, 1977), los profesores pueden adquirir «conocimiento sistemático y exacto sobre su propia enseñanza» (Uusikylä, I977, p. 89*). Al colocar al profesor tanto detrás como delante de la tecnología de observación, este (el profesor) es construido como ambos, el sujeto y el objeto de la investigación, acentuando así el doble trabajo sobre el ser que debe ser realizado como una personalidad del docente. El sujeto docente consciente debe llevar a cabo su enseñanza de una manera creativa, atenta y deliberativa, así como reflexionar sobre sus acciones personales para resolver problemas y tomar decisiones de manera más efectiva.

Una vez más, este razonamiento contiene la alquimia: una disolución de cualquier diferencia entre una situación de investigación y una situación de enseñan$\mathrm{za}$, entre lo que supuestamente es definido como «investigación propiamente dicha» $\mathrm{y}$ «el pensamiento de pedagogos practicantes» (Koskenniemi, 1967, p. 165*), entre el investigador-científico y el profesor:

Cada situación de planificación y enseñanza puede convertirse en una situación de investigación. En tal caso, la acción del profesor es independiente y no necesita simplemente confiar en el libro de texto o en las autoridades. A medida que se desarrolla la actitud de un investigador, también lo hace la personalidad del docente y su actitud personal hacia su práctica. (Malinen, 1974a, p. 350*)

La noción de alquimia de Popkewitz ayuda a hacer visible, entonces, cómo la «investigación» se moviliza como una «intervención pedagógica» (Popkewitz,

12 Una nota sobre la traducción: «las leyes naturales» deben entenderse aquí en el mismo sentido que «la ley natural de la gravedad» o «las leyes naturales de la perspectiva» en lugar de como un término filosófico con respecto a la teoría moral. 

JOHANNA SITOMANIEMI-SAN

2002, p. 264), una estrategia de gobierno con tecnologías particulares para trabajar sobre el ser del profesor y regular su autonomía. La fabricación del profesor como un investigador y un pensador científico implica una estrategia a través de la cual programar la manera en que el profesor debe pensar, actuar, sentir y ver en el aula -y cómo debe saber y trabajar sobre sí mismo-. Desde esta perspectiva, pueden no parecer tan evidentes aquellos argumentos que sugieren que la inserción de la ciencia en la formación docente ha provocado un cambio desde el ethos vocacional a un ethos científico y ha transformado a las «velas de la nación» en expertos científicos.

Si bien se cita raras veces a Koskenniemi en la literatura actual sobre la formación del profesorado basada en la investigación, la influencia koskenniemiana en la formación finlandesa del profesorado continúa siendo efectiva, como también han señalado Saari et al. (20I4). La efectividad de la línea de razonamiento DPA es visible en las articulaciones actuales en las que el profesorado debe «pensar como un investigador», tener «una actitud inquieta» y, por lo tanto, "observar, analizar y desarrollar su trabajo» (Toom et al., 20I0, p. 339). En la literatura, se describe y define que la formación del profesorado basada en la investigación presenta el objetivo de "promover las capacidades y la toma de decisiones didácticas del profesorado y de inspirarlo a adoptar un enfoque de investigación en su trabajo diario» (Aspfors y Eklund, 20I7, p. 403). Las «tradiciones de investigación cualitativas y cuantitativas» son explicadas como incluidas en el currículum de la formación del profesorado con el objetivo de «formar a los estudiantes para encontrar y analizar problemas que pueden esperar enfrentar en su trabajo futuro» (Niemi, 2oI2, p. 32). Quizás, irónicamente, es la inserción de la ciencia empírica -y la centralidad de la metodología de investigación en esta inserción- lo que viene a mantener el énfasis sobre el carácter, las actitudes y la personalidad del docente. La metodología de investigación y la «orientación investigadora» sirven para la creación de un agente autorreflexivo: observar y observarse a sí mismo es volver la mirada científica hacia uno mismo, conocerse a sí mismo y demostrar la personalidad de uno mismo en el proceso.

Por lo tanto, a pesar del cambio explícito de lo especulativo a lo científico y, por consiguiente, a lo racional y a lo que podría ser argumentado como técnico, la cientifización de la formación finlandesa del profesorado también puede percibirse como una narrativa de lo contrario: la formación del profesorado académica y basada en la investigación es concebida como emancipadora del profesor respecto al tecnicismo y la subordinación, conduciéndolo hacia el crecimiento interno y la libertad. La presencia de la «investigación» y la «ciencia» en la formación del profesorado contribuye a la fabricación y el gobierno del profesor como un sujeto autoconsciente, reflexivo, deliberativo, analítico, solucionador de problemas y creativo. Si bien el discurso de la formación del profesorado científica y basada en la investigación compone una lista (no completamente) diferente de cualidades y disposiciones ideales para el profesorado en comparación con aquella producida por Haavio durante la década de I940, se ha mantenido, sin embargo, es la centralidad de la búsqueda de fabricar y cultivar la «personalidad del docente». 
DE LA PERSONALIDAD DOCENTE A LA METODOLOGÍA DE INVESTIGACIÓN: HISTORIZANDO 278 LA FORMACIÓN DEL PROFESORADO BASADA EN LA INVESTIGACIÓN EN FINLANDIA JOHANNA SITOMANIEMI-SAN

\section{Conclusión: repensando la historia de la formación finlandesa del profesorado}

En este artículo se ha pensado con la noción foucaultiana de gubernamentalidad (Burchell, Gordon y Miller, 1991; Miller y Rose, 2008; Rose, 1999), junto con la noción de alquimia de Popkewitz, para revisar la reforma de los años 70 que «cientificó» la formación finlandesa del profesorado. Lo que se sugiere a través del análisis es que es a través de la alquimia de la investigación (y la ciencia) como se fabrica y gobierna al profesor de la formación del profesorado basada en investigación, más que a través de la investigación en sí misma ${ }^{13}$. Es decir, la metodología de investigación se transforma en una tecnología para gobernar y trabajar sobre el ser del profesor; para instalar disposiciones, mentalidades y autoconciencia. La noción de personalidad docente es fundamental para este esfuerzo de gobierno: en lugar de ser desplazada en el «nuevo» ethos científico de la formación finlandesa del profesorado, se reconfigura como ambas, como un objetivo y como parte de la tecnología a través de la cual el profesor es producido como un investigador-científico, solucionador de problemas y practicante reflexivo.

Esta observación ofrece una forma alternativa de pensar sobre el pasado y el presente de la formación finlandesa del profesorado. Sugiere que la transformación de la formación del profesorado finlandesa desde un ethos especulativo-profesional a uno científico-profesional quizás no sea tan sencilla como podría parecer a primera vista; que la cientificización de la formación finlandesa del profesorado tiene efectos sobre las subjetividades docentes, los cuales exceden a lo que con mayor frecuencia se ha enmarcado como la construcción del profesor como un «experto científico» o como «gobernado por la ciencia». Contrariamente al argumento de Heikkinen (discutido en la «Introducción»), es posible argumentar que la noción y el ideal de la personalidad docente no se perdieron en la cientificización de la formación del profesorado y en la construcción del profesor como investigador. En lugar de suponer que la centralidad de la personalidad docente ha sido reemplazada por la de la metodología de investigación, es posible sugerir que la presencia de la metodología de investigación crea las condiciones para rearticular el énfasis en la personalidad docente y, por lo tanto, para reinscribir el orden moral-nacional de las cosas en el presente educativo.

De esta manera, el esfuerzo genealógico e historizador de este artículo para «conectar los puntos» entre la personalidad docente y la metodología de la investigación apunta -quizás demasiado tenuemente- a una configuración particular de humanismo y nostalgia, de la que parece depender el discurso finlandés de la formación del profesorado basada en la investigación. Es un discurso en el que la formación del profesorado académica y científica se recuerda a sí misma una

${ }^{13}$ El propósito aquí no es esencializar o fijar el significado de la investigación o la ciencia. Es simplemente llamar la atención sobre la alquimia que transforma la investigación intelectual y disciplinaria en un conjunto de prácticas a través de las cuales gobernar al sujeto: «Solo el homónimo aparece, como una placa ubicua para marcar una casa» (POPKEWITZ, 2002, p. 262). 
y otra vez que no es una empresa técnica sino un proyecto de bildung y cultivo: para el ser del profesor (Heikkinen, I999; Heikkinen, Syrjäläinen, Syrjäläinen y Värri, 2008), para la nación (Krokfors, 2005; Uusikylä, 1992) y para un mundo global (Niemi, 2010).

\section{Agradecimientos}

Quisiera expresar mi agradecimiento a Thomas Popkewitz por los útiles comentarios sobre los borradores anteriores de este estudio y por las conversaciones sobre (y más allá de) la formación del profesorado. También me gustaría agradecer a Erika Bullock, Gail Prasad y Sara Torres por los espacios que compartieron conmigo y que me permitieron escribir.

\section{Bibliografía}

Alikoski, J.: «FYTT ja opettajankoulutus» [«FYTT (El Consejo de Filosofía y Ciencias Sociales) y la educación del profesorado»], Kasvatus [Educación], 4(4) (1973), pp. 212-216.

AsPFORS, J. y EKLUND, G.: «Explicit and implicit perspectives on research-based teacher education: Newly qualified teachers' experiences in Finland», Journal of Education for Teaching, (2017), pp. I-I4.

Autio, T.: Subjectivity, curriculum and society: Between and beyond German Didaktik and Anglo-American curriculum studies, Mahwah, NJ, Lawrence Erlbaum Associates, 2006.

Autio, T.: «The internationalization of curriculum research», en PINAR, W. F. (ed.): International handbook of curriculum research, New York y London, Routledge, 20I4, (Second edition ed.), pp. I7-3I.

Burchell, G.; Gordon, C. y Miller, P. (eds.): The Foucault effect: Studies in governmentality with two lectures by and an interview with Michel Foucault, London, Harvester Wheatsheaf, I991.

DARLing-Hammond, L.: «Teacher education around the world: What can we learn from international practice?», European Journal of Teacher Education, 40(3) (2017), pp. 29I-309.

Foucault, M.: «Nietzsche, genealogy, history», en Rabinow, P. (ed.): The Foucault reader: An introduction to Foucault's thought, London, Penguin Books, I991, pp. 76-100.

HaAvio, M.: Opettajapersoonallisuus [Personalidad Docente], Jyväskylä, Gummerus, 1948.

HaAvio, M.: «Seminaarihenki» ["El espíritu de seminario»], Kasvatus ja Koulu [Educación y Escuela], 5I(6) (1965), pp. 286-291.

Hammersley, M.: «On the teacher as researcher», Educational Action Research, I(3) (1993), pp. $425-445$.

Hansén, S. y EkLund, G.: «Finnish teacher education: Challenges and possibilities», Journal of International Forum of Educational Research, I(2) (2014), pp. I-I2.

Heik Kinen, H. L. T.: «Opettajapersoonan uusi tuleminen» [«El resurgir de la personalidad docente»], en NiEMI, H. (ed.): Opettajankoulutus modernin murroksessa [La formación del profesorado en la ruptura de la modernidad], Tampere, TAJU, I999, pp. I80-I9I.

Heikkinen, H. L. T.; SYrJäläinen, E.; SyrJäläinen, E. y VÄrRI, V.: «Opettajankoulutuksesta elämänmittaiseen» [«Desde la formación del profesorado al crecimiento a lo largo de toda la vida»], Kasvatus [Educación], 39(3) (2008), pp. 27I-276. 
Horlacher, R.: The educated subject and the German concept of Bildung: A comparative cultural history, New York, Routledge, 2016.

HYtÖNEN, J.: «Kasvattajan pitää osata ajatella» [«El educador debe saber cómo pensar»], Kasvatus [Educación], 6(6) (1977), pp. I07-I09.

Jakku-Sihvonen, R. y Niemi, H.: "The Bologna process and its implementation in teacher education», en JAKKU-SiHVONEN, R. y Niemi, H. (eds.): Research-based teacher education in Finland: Reflections by Finnish teacher educators, Turku, Finnish Educational Research Association, 2006a, pp. 17-29.

Jakku-Sinvonen, R. y Niemi, H. (eds.): Research-based teacher education in Finland: Reflections by Finnish teacher educators, Turku, Finnish Educational Research Association, 20o6b.

KaikKonen, M.: «Kasvattajan pitää osata» [«El educador de saber cómo hacer»], Kasvatus [Educación], 8(2) (1977), pp. I05-I06.

Kansanen, P.: «Research-based teacher education», en JAKKu-Sinvonen, R. y Niemi, H. (eds.): Education as a societal contributor: Reflections by Finnish educationalists, Frankfurt am Main, Peter Lang, 2007, pp. I3I-I46.

Kivinen, O. y Rinne, R.: «Opettajankoulutus keskusteluun» [«Debatiendo sobre la formación del profesorado»], Kasvatus [Educación], 20(5) (1989), pp. 418-422.

Komulainen, E.: «Didaktisen prosessianalyysin (DPA-Helsinki) hankkeen alkuvaiheesta» [«Sobre el estado inicial del proyecto DPA-Helsinki»], Didacta Varia, I4(I) (2009), pp. 5I-57.

Koskenniemi, M.: «Opettajanvalmistus ja yliopisto» [«La preparación del profesorado y la universidad»], Kasvatusopillinen Aikakauskirja [Revista de Educación], 99(2) (1962), pp. 49-52.

Koskenniemi, M.: «Kasvatuksen teoria ja opettajanvalmistus» [ «Teoría educativa y formación del profesorado»], en Valtasaari, A.; Henttonen, A.; Järvi, L. y Nurmi, V. (eds.): Kansakoulu i866-1966 [La Escuela Popular 1866-1966], Helsinki, Otava, 1966, pp. 237-240.

KoskenNiemi, M.: «Tutkimus ja käytäntö kasvatuksen maailmassa» [«Investigación y práctica en el mundo de la educación»], Kasvatusopillinen Aikakauskirja [Revista de Educación], I04(4) (1967), pp. 165-167.

Koskenniemi, M.: Opetuksen teorian perusaineksia [Los componentes básicos de la teoría de la enseñanza], Helsinki, Otava, 1968.

Koskenniemi, M. y Hälinen, K.: Didaktiikka: Läbinnä peruskoulua varten [Didácticas: principalmente para el contexto de las escuelas primarias], Helsinki, Otava, 1970.

Koski, L.: "The changing moral orders of educational stories in ABC books», en ANTIKAINEN, A. (ed.): Transforming a learning society: The case of Finland, Bern, Peter Lang, 2005, pp. $33-52$.

Koski, L. y Filander, K.: «Transforming causal logics in Finnish adult education: Historical and moral transitions rewritten», International Journal of Lifelong Education, 32(5) (2013), pp. 583-599.

KROKFORS, L.: «Vaikuttajaopettaja: Eettinen ja kriittinen päätöksentekijä» [«El professor influyente: el tomador de decisiones ético y crítico»], en LuUkKainen, O.y Valli, R. (eds.): Kaksitoista teesiä opettajalle [Doce tesis para el profesorado], Jyväskylä, PS-kustannus, 2005, pp. 67-78.

KYÖsтіÖ, O. K.: «Opettajanvalmistuksen yhtenäistämisestä» [«Sobre la unificación de la preparación del profesorado»], Kasvatusopillinen Aikakauskirja [Revista de Educación], IO2(I) (1965), pp. 5-10.

LABAREE, D. F.: «Power, knowledge, and the rationalization of teaching: A genealogy of the movement to professionalize teaching», Harvard Educational Review, 62(2) (1992), pp. I23-I54.

MaARANEN, K. y KROKFORS, L.: «Researching pupils, schools and oneself: Teachers as integrators of theory and practice in initial teacher education", Journal of Education for Teaching, 34(3) (2008), pp. 207-222. 
DE LA PERSONALIDAD DOCENTE A LA METODOLOGÍA DE INVESTIGACIÓN: HISTORIZANDO LA FORMACIÓN DEL PROFESORADO BASADA EN LA INVESTIGACIÓN EN FINLANDIA JOHANNA SITOMANIEMI-SAN

Malinen, P.: «Opettaja tutkijana» [«El professor como investigador»], Kasvatus, 5(6) (1974a), pp. 349-35I.

Malinen, P.: Opettajankoulutus akateemisten opintojen osana: Analyysi opettajankoulutuksen tieteellisyydestä sekä tähän koulutukseen liittyvän tutkimustoiminnan luonteesta [La formación del profesorado como un componente de los estudios académicos: un análisis de la cientificidad de la formación del profesorado y de la naturaleza de la formación del profesorado -las actividades de investigación relacionadas hasta la fecha], Jyväskylä, University of Jyväskylä, 1974b.

Miller, P. y Rose, N.: Governing the present: Administering economic, social and personal life, Cambridge, Polity Press, 2008.

NiEmI, H.: «Suomalainen opettajankoulutus uusien haasteiden edessä» [«La formación finlandesa del profesorado frente a nuevos retos»], en Kallioniemi, A.; Toom, A.; Ubani, M. y LiNNANSAARI, H. (eds.): Akateeminen luokanopettajakoulutus: 30 vuotta teoriaa, käytäntöä ja maistereita [La formación del profesorado de clase académica: 30 años de teoría, práctica y másteres], Turku, The Finnish Educational Research Association, 2010, pp. 27-50.

Niemi, H.: «The societal factors contributing to education and schooling in Finlan», en Niemi, H.; Toom, A. y Kallioniemi, A. (eds.): Miracle of education: The principles and practices of teaching and learning in Finnish schools, Rotterdam, Sense Publishers, 2012, pp. 19-38.

Popkewitz, T.: Struggling for the soul: The politics of schooling and the construction of the teacher, New York, Teachers College Press, 1998.

POPKEWITZ, T.: «How the alchemy makes inquiry, evidence, and exclusion», Journal of Teacher Education, 53(3) (2002), pp. 262-267.

Popkewitz, T.: «Inventing the modern self and John Dewey: Modernities and the traveling of pragmatism in education - an introduction», en POPKEWITZ, T. (ed.): Inventing the modern self and John Dewey: Modernities and the traveling of pragmatism in education, New York, Palgrave Macmillan, 2005, pp. 3-36.

Popkewitz, T.: «Alchemies and governing: Or, questions about the questions we ask», Educational Philosophy and Theory, 39(I) (2007), pp. 64-83.

Popkewitz, T.: «The limits of teacher education reforms: School subjects, alchemies, and an alternative possibility», Journal of Teacher Education, 6I(5) (2010), pp. 413-42I.

Popkewitz, T. (ed.): Rethinking the history of education: Transnational perspectives on its questions, methods, and knowledge, New York, Palgrave Macmillan, 2013.

Popkewitz, T.; Franklin, B. M. y Pereyra, M. A. (eds.): Cultural history and education: Critical essays on knowledge and schooling [Historia cultural y educación: ensayos críticos sobre el conocimiento y la escolarización], New York, Routledge, 200I.

Rose, N.: Governing the soul: The shaping of the private self (2nd ed.), London, Free Association Books, 1999.

SAARI, A.: Kasvatustieteen tiedontabto: Kriittisen historian näkökulmia suomalaiseen kasvatuksen tutkimukseen [Educación y el deseo de conocimiento: una historia crítica de la bistoria de la investigación educativa en Finlandia], Turku, The Finnish Educational Research Association, 20II.

SAARI, A.: «Suomalaisen koulupedagogiikan kasvatustieteellinen perusta I90o-luvulla» [«Las bases de las ciencias educativas de la pedagogía escolar finlandesa en los i9oos»], Kasvatus E Aika [Educación y Tiempo], 7(4) (2013), pp. 72-78.

SaAri, A.; Salmela, S. y Vilkkilä, J.: «Governing autonomy: Subjectivity, freedom, and knowledge in Finnish curriculum discourse», en PINAR, W. F. (ed.): International handbook of curriculum research (2nd ed.), New York y London, Routledge, 20I4, pp. 183-200.

SAHLBERG, P.: Finnish lessons 2.0: What can the world learn from educational change in Finland? (2nd ed.), New York, Teachers College Press, 20I5. 
SalomaA, J. E.: «Kasvattajan olemuksesta» [«Sobre el ser del educador»], Kasvatus ja Koulu, 33 (1947), pp. 3-18.

Säntti, J.; Rantala, J.; Salminen, J. y Hansen, P.: «Bowing to science: Finnish teacher education turns its back on practical schoolwork», Educational Practice and Theory, 36(I) (20I4), pp. 2I-4I.

SilANDER, T. y VÄLIJÄRVI, J.: «The theory and practice of building pedagogical skill in Finnish teacher education", en Meyer, H. y Benavot, A. (eds.): PISA, power, and policy: The emergence of global educational governance, Oxford, Symposium Books, 2013, pp. 77-97.

Simola, H.: «Educational science, the state, and teachers: Forming the corporate regulation of teacher education in Finland», en POPKEWITZ, T. (ed.): Changing patterns of power: Social regulation and teacher education reform, Albany, State University of New York Press, 1993, pp. I6I-2IO.

Simola, H.: «Constructing a school-free pedagogy: Decontextualization of Finnish state educational discourse», Journal of Curriculum Studies, 30(3) (1998a), pp. 339-356.

Simola, H.: «Decontextualizing teachers' knowledge: Finnish didactics and teacher education curricula during the 1980 s and 1990s", Scandinavian Journal of Educational Research, 42(4) (1998b), p. 325 .

Simola, H.: «Firmly bolted into the air: Wishful rationalism as a discursive basis for educational reforms», Teachers College Record, 99(4) (1998c), pp. 731-757.

Simola, H.; Kivinen, O. y Rinne, R.: «Didactic closure: Professionalization and pedagogic knowledge in Finnish teacher education», Teaching and Teacher Education, 13(8) (1997), pp. 877-89I.

Sitomaniemi-SAn, J.: Fabricating the teacher as researcher: A genealogy of academic teacher education in Finland, Acta Universitatis Ouluensis, EI57, Oulu, University of Oulu, 2015.

Toom, A.; Kynäslahti, H.; Krokfors, L.; Jyrhämä, R.; Byman, R.; Stenberg, K., ... KansANEN, P.: «Experiences of a research-based approach to teacher education: Suggestions for future policies», European Journal of Education, 45(2) (2010), pp. 331-344.

TröHler, D.: «The discourse of German Geisteswissenschaftliche Pädagogik - A contextual reconstruction», Paedagogica Historica, 39(6) (2003), pp. 759-778.

TröHler, D.: Languages of education: Protestant legacies, national identities, and global aspirations, New York, Routledge, 20II.

UUSIKYLÄ, K.: «Tulevien opettajien kouluttamisesta didaktiseen ajatteluun» [«Sobre la educación de futuros profesores hacia el pensamiento didáctico»], Kasvatus [Educación], 8(2) (1977), pp. 87-90.

UusIKYLÄ, K.: Miten kuvaan opetustapabtumaa [Cómo describir el evento de enseñanza], Helsinki, Gaudeamus, I980.

UUSIKYLÄ, K.: «Tarvitaanko akateemista luokanopettajankoulutusta?» [«¿Es necesaria la formación del profesorado de clase académica?»], Kasvatus [Educación], 23(I) (1992), pp. 57-6I.

VilJANEN, E.: «Tutkimuksen rooli opettajankasvatuksessa» [«El papel de la investigación en la formación de profesores»], Kasvatus [Educación], Io(4) (1979), pp. 216-223.

Viljanen, E.: «Mallikouluista tutkimuskouluihin» [«Desde escuelas modelo a escuelas de investigación»], en KarI, J. (ed.): Tutkimuspohjaista koulutusta kohti: Professori Veikko Heinosen jublakirja 7.5.1988 [Hacia una educación basada en la investigación: escritos en honor al Professor Veikko Heinonen, 7 de mayo de 1988], Jyväskylä, Research Institute for Educational Sciences, 1988, pp. 19-32.

Westbury, I.; Hansén, S.; Kansanen, P. y Björkvist, O.: «Teacher education for research-based practice in expanded roles: Finland's experience», Scandinavian Journal of Educational Research, 49(5) (2005), pp. 475-485.

Westbury, I.; Hopmann, S. y Riquarts, K. (eds.): Teaching as a reflective practice: The German Didaktik tradition, Mahwah (N. J.), Lawrence Erlbaum Associates, 2000. 